10.2

\title{
Акустоэлектрический преобразователь на основе электрокинетического явления потенциал течения
}

\author{
(С) Б.П. Шарфрарец ${ }^{1}$, С.П. Дмитриев ${ }^{1}$, В.Е. Курочкин ${ }^{1}$, Ф.Ф. Легуша ${ }^{2}$ \\ ${ }^{1}$ Институт аналитического приборостроения РАН, Санкт-Петербург, Россия \\ ${ }^{2}$ Санкт-Петербургский государственный морской технический университет, Санкт-Петербург, Россия \\ E-mail: sharb@mail.ru
}

Поступило в Редакцию 21 июля 2021 г.

В окончательной редакции 21 июля 2021 г.

Принято к публикации 14 сентября 2021 г.

\begin{abstract}
Предложена математическая модель акустоэлектрического преобразователя, основанного на использовании электрокинетического явления потенциал течения. Теоретически показано, что потенциал течения растет пропорционально величине напряженности постоянного электрического поля накачки, что подтверждено экспериментально. Описываемый преобразователь обладает переменной чувствительностью. Экспериментально получены достаточно большие значения чувствительности электрокинетического микрофона. Полученные результаты могут быть использованы при конструировании акустоэлектрических преобразователей.
\end{abstract}

Ключевые слова: акустоэлектрическое преобразование, электрокинетические явления, потенциал течения, гидродинамика потенциала течения, накачка энергии, чувствительность электрокинетического микрофона.

DOI: 10.21883/PJTF.2021.24.51794.18970

Ранее в работах [1-3] рассмотрено электроакустическое преобразование, основанное на электрокинетическом явлении (ЭЯ) электроосмоса. Основное отличие от существовавших ранее подобных преобразований (см., например, работу [4]) состоит в использовании режима накачки энергии акустических колебаний за счет энергии дополнительно приложенного к пористой среде постоянного электрического поля.

В настоящей работе рассматривается акустоэлектрическое преобразование, основанное на ЭЯ потенциал течения также при наличии процесса накачки. Без режима накачки эта проблема рассмотрена в работе $[4, \S 3]$. При наличии накачки в процессе акустоэлектрического преобразования используются оба ЭЯ: и электроосмос, и потенциал течения. Впервые теоретически с учетом процесса накачки этот вопрос был частично рассмотрен в [5] и других работах авторов. Здесь кратко приводятся теоретические аспекты, изложенные в этих работах, а также новые результаты по рассматриваемой теме.

Акустоэлектрическое преобразование основано на ЭЯ потенциал течения - явлении возникновения разности потенциалов на торцах капилляра (пористого тела) при течении в нем жидкости. Основную роль в этом играют двойной электрический слой, формирующийся на границе раздела фаз, и его поляризация. Для упрощения рассматривают это явление в цилиндрическом капилляpe, электрокинетические процессы в котором подобны процессам в обычной пористой структуре при не очень обременительных допущениях $[6, \S 2.2]$. Ось капилляра далее полагаем ориентированной вдоль оси $0 z$.

Процесс образования разности потенциалов $\Delta \varphi$ на торцах капилляра, наполненного жидкостью, при подаче акустического поля подробно описан, например, в рабо- те $[6, \S 2.5]$. Выражение, связывающее величину потенциала течения на торцах капилляра $\Delta \varphi$ (т.е. разность потенциалов) с величиной перепада давления на них $\Delta p$, имеет вид $[6$, с. $10 ; 7$, с. 516$]$ :

$$
\Delta \varphi=-\varepsilon \varepsilon_{0} \tilde{\zeta} \Delta p / \eta \sigma .
$$

Здесь $\sigma$ - удельная проводимость жидкости, $\varepsilon, \varepsilon_{0}-$ соответственно диэлектрическая проницаемость жидкости и электрическая постоянная, $\tilde{\xi}$ - дзета-потенциал, $\eta$ - динамическая вязкость жидкости. Соотношение (1) показывает, что потенциал течения не зависит от площади сечения капилляра, а задается только величиной перепада давления $[7$, с. 516]. При переходе к реальной связнодисперсной системе возникают усложнения, однако описанная закономерность (1) остается справедливой и в этом случае $[8$, с. 184$]$.

В (1) величины $\Delta \varphi$ и $\Delta p$ не зависят от времени и представляют собой разности соответствующих величин на концах капилляра. В случае их зависимости от времени и координаты $z$ на оси капилляра в [5] получено уравнение для функций $\varphi(z, t)$ и $p(z, t)$

$$
-\rho_{e} \partial \varphi / \partial z=\left(\rho_{e} \varepsilon \varepsilon_{0} \tilde{\xi} / \eta \sigma\right) \partial p / \partial z,
$$

где $\rho_{e}$ - объемная плотность электрического заряда в электролите. Пусть $\mathbf{E}=-\nabla \varphi-$ значение вектора электрической напряженности, соответствующее потенциалу течения. Из (2) с учетом допущения $\mathbf{E}=(0,0, E)$ для гармонических процессов $\mathbf{E}(z) \exp (-i \omega t)$ и $p \exp (-i \omega t)$ справедливо выражение [5]:

$$
\mathbf{F}=\rho_{e} \mathbf{E}(z)=\left(\rho_{e} \varepsilon \varepsilon_{0} \tilde{\xi} / \eta \sigma\right) \partial p / \partial z,
$$

т. е. в случае потенциала течения возникает действующая на жидкость объемная сила $\mathbf{F}$ (3). 
Механизм процесса накачки с помощью анализа уравнения Навье-Стокса применительно к акустоэлектрическому преобразованию, когда к торцам капилляра, наполненного жидкостью, одновременно прикладывается постоянное электрическое поле $\mathbf{E}_{0}$ и внешнее акустическое поле с давлением $p_{a}$, приведен в [5]. После выделения линейной (акустической) части из исходного нелинейного уравнения Навье-Стокса получается следующее линейное уравнение относительно акустических полей $(\mathbf{v}, p)$

$$
\begin{aligned}
\rho_{0}(\partial \mathbf{v} / \partial t & \left.+\left(\mathbf{v}_{0} \cdot \nabla\right) \mathbf{v}+(\mathbf{v} \cdot \nabla) \mathbf{v}_{0}\right)=-\nabla p+\eta \Delta \mathbf{v} \\
& +(\xi+\eta / 3) \nabla \nabla \cdot \mathbf{v}+\mathbf{F} .
\end{aligned}
$$

Здесь $\mathbf{v}_{0}$ - поле скорости, вызванное стационарным электрическим полем $\mathbf{E}_{0} ; \rho_{0}-$ стационарная плотность жидкости; $\xi$ - объемная вязкость; F - объемная сила, которая определена в (3). К уравнению движения (4) следует добавить стандартное линеаризованное уравнение непрерывности для сжимаемой жидкости $\frac{\partial \rho}{\partial t}+\rho_{0} \nabla \cdot \mathbf{v}=0$.

Для упрощения уравнения (4) принимаем допущение о малой толщине двойного слоя на границе раздела жидкости и внутренней поверхности капилляра, что определяется неравенством $\kappa a>>1(\kappa=1 / \lambda ; \lambda-$ толщина двойного слоя; $a-$ радиус капилляра). В этом случае скорость электроосмотического движения жидкости практически во всем сечении капилляра равна скорости осмотического движения Гельмгольца-Смолуховского $U_{e o}[6$, c. 10$]$ :

$$
U_{e o}=E_{0} \varepsilon \varepsilon_{0} \tilde{\xi} / \eta=\text { const. }
$$

Таким образом, имеем в цилиндрической системе координат $\mathbf{v}_{0}=\left(0,0, U_{e o}\right)$, что приводит к выражению $\left(\mathbf{v}_{0} \cdot \nabla\right) \mathbf{v}=U_{e o} \partial \mathbf{v} / \partial z[9, \mathbf{c .} 68,83]$ или окончательно с учетом (5)

$$
(\mathbf{v} \cdot \nabla) \mathbf{v}=U_{e o} \partial \mathbf{v} / \partial z=E_{0}\left(\varepsilon \varepsilon_{0} / \eta\right) \tilde{\zeta} \partial \mathbf{v} / \partial z .
$$

Перепишем (4) с учетом последнего равенства, а также очевидного равенства $\nabla \mathbf{v}_{0} \equiv 0$.

$$
\begin{aligned}
\rho_{0} \partial \mathbf{v} / \partial t & =-\nabla p+\eta \Delta \mathbf{v}+(\xi+\eta / 3) \nabla \nabla \cdot \mathbf{v} \\
& -\rho_{0} U_{e o} \partial \mathbf{v} / \partial z
\end{aligned}
$$

Полагая процесс потенциальным $\mathbf{v}=\nabla \Phi$, аналогично [3] приводим (6) к скалярному виду (с учетом возможности здесь коммутации операторов $\partial / \partial z \nabla=\nabla \partial / \partial z[9, \mathrm{c.84}])$

$$
\rho_{0} \partial \Phi / \partial t=-p+(\xi+4 \eta / 3) \Delta \Phi-\rho_{0} U_{e o} \partial \Phi / \partial z .
$$

В гармоническом случае с временны́м фактором $\exp (-i \omega t)$, сохраняя те же обозначения для амплитуд, для амплитуды потенциала $\Phi$ из (7) получаем уравнение Гельмгольца

$$
\Delta \Phi+k^{2} \Phi=\left(k^{2} / i \omega\right) U_{e o} \partial \Phi / \partial z,
$$

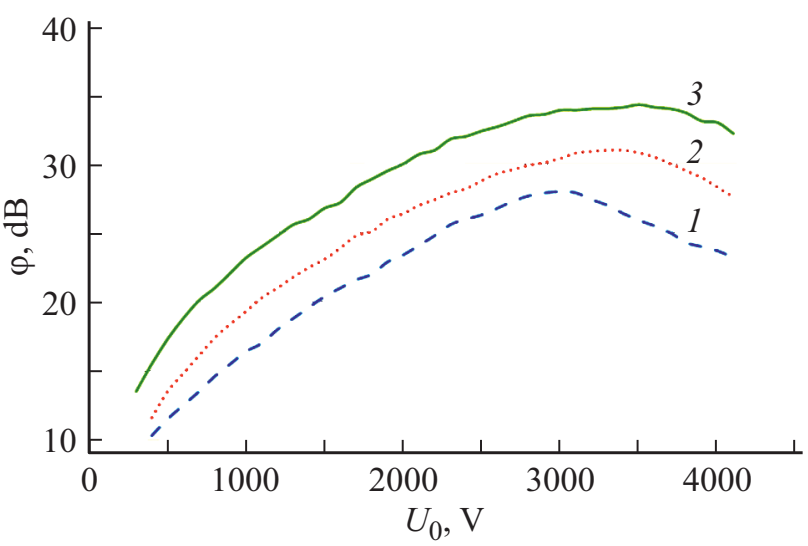

Зависимости потенциала течения $\varphi$ в приемнике от величины напряжения накачки $U_{0}$ при значениях давления акустического поля $p_{a i}=85(1), 90$ (2) и 95 (3).

$k^{2}=\left(\omega^{2} / c^{2}\right) \rho_{0} /\left(\left(\rho_{0}-i \omega / c^{2}\right)(\xi+4 \eta / 3)\right)$ - квадрат соответствующего волнового числа.

Уравнение (8) - неоднородное уравнение Гельмгольца относительно амплитуды потенциала $\Phi$ скорости $\mathbf{v}$. Из (8) следует, что с ростом величины скорости $U_{e o}$ должна расти величина амплитуды $\Phi$, а следовательно, и величины амплитуд $\mathbf{v}$ и $p$, так как имеют место зависимости $\mathbf{v}=\nabla \Phi, p=\left(\rho_{0} c^{2} / i \omega\right) \Delta \Phi$. Анализ в [5] показывает, что с ростом скорости электроосмотического течения растет и величина потенциала течения.

Для проверки теории были проведены натурные эксперименты. В качестве приемника использовалась расположенная в воздушной среде матрица - пачка офисной бумаги формата A4 толщиной $2.5 \mathrm{~mm}$, помещенная между двумя перфорированными алюминиевыми пластинами-электродами. К электродам матрицы подводилось электрическое поле $\mathbf{E}_{0}$. С электродов снималось переменное напряжение, образуемое в матрице под воздействием внешнего звукового поля. В первом эксперименте изучалось влияние накачки на величину потенциала течения. На частоте $f=2 \mathrm{kHz}$ динамик на фиксированном от матрицы расстоянии поочередно излучал акустическое давление $p_{a}$ трех различных фиксированных на апертуре матрицы уровней $p_{a i}=85,90$, $95 \mathrm{~dB}(i=1,2,3)$ относительно уровня $20 \mu \mathrm{Pa}$. При каждом фиксированном давлении измерялась зависимость величины потенциала течения $\varphi_{i}$ от потенциала $U_{0}$ электрического поля $E_{0}: \varphi_{i}=f\left(p_{a i}, U_{0}\right)\left(\left|E_{0}\right|=\left|U_{0}\right| / d\right)$, где $d$ - толщина слоя бумаги). Результаты измерений представлены на рисунке. Кривые 1, 2 и 3 отвечают величине давления на апертуре 85, 90 и $95 \mathrm{~dB}$ соответственно. Величина потенциала течения $\varphi$ в зависимости от $U_{0}$ измерялась в относительных единицах. Из рисунка видно схожее поведение кривых во всех трех случаях. Сначала потенциал течения растет пропорционально $U_{0}$, затем наблюдается зона насыщения, и, наконец, с ростом $U_{0}$ возникает спад потенциала течения $\varphi$. Объясняется это возникновением турбулентного режима движения жид- 
Зависимость чувствительности от накачки $U_{0}$ при давлении звука $p=0.893 \mathrm{~Pa}$

\begin{tabular}{c|c|c}
\hline $\begin{array}{c}\text { Накачка } U_{0}, \\
\mathrm{~V}\end{array}$ & $\begin{array}{c}\text { Потенциал } \\
\text { течения } \varphi, \\
\mathrm{mV}\end{array}$ & $\begin{array}{c}\text { Чувствитель- } \\
\text { ность, } \\
\mathrm{mV} / \mathrm{Pa}\end{array}$ \\
\hline 0 & 1189 & 1330 \\
132 & 1603 & 1795 \\
397 & 2018 & 2259 \\
529 & 2540 & 2841 \\
661 & 2851 & 3191 \\
1322 & 4027 & 4507
\end{tabular}

кости при некоторых значениях $U_{0}$ в пористой структуре матрицы. Из рисунка также видно, что чувствительность преобразователя при фиксированном акустическом поле $p_{a i}$ является величиной переменной и зависит от $U_{0}$. Во втором эксперименте были рассчитаны величины чувствительности преобразователя. Звук частотой $1 \mathrm{kHz}$ имел уровень давления на плоскости преобразователя $93 \mathrm{~dB}$. Чувствительность была замерена при различных уровнях накачки. Результаты измерений сведены в таблицу.

В результате проведенных исследований предложены физическая и математическая модели функционирования приемника, основанного на использовании ЭЯ потенциал течения. Теоретически показано и экспериментально подтверждено, что потенциал течения возрастает пропорционально величине электрического поля накачки. Экспериментально выявлено наличие насыщения в этом процессе, обусловленного нелинейностью процесса и возникновением режима турбулентного движения жидкости в теле преобразователя.

Чувствительность электрокинетического микрофона оказалась существенно выше, чем у наиболее чувствительных угольного и конденсаторного микрофонов: 200-400 и $10 \mathrm{mV} /$ Ра соответственно [10, с. 152].

Результаты работы могут быть использованы в теории и практике конструирования обратимых электроакустических и акустоэлектрических преобразователей.

\section{Финансирование работы}

Работа выполнена в ИАП РАН в рамках государственного задания 075-00780-21-00 по теме № 0074-2021-0013 Министерства науки и высшего образования.

\section{Конфликт интересов}

Авторы заявляют, что у них нет конфликта интересов.

\section{Список литературы}

[1] В.А. Сергеев, Б.П. Шарфарец, Науч. приборостроение, 28 (2), 25 (2018).
[2] В.Е. Курочкин, В.А. Сергеев, Б.П. Шарфарец, Ю.В. Гуляев, ДАН, 483 (3), 265 (2018).

[3] Б.П. Шарфарец, В.Е. Курочкин, В.А. Сергеев, Ю.В. Гуляев, Акуст. журн., 66 (4), 453 (2020).

[4] М.С. Касимзаде, Р.Ф. Халилов, А.Н. Балашов, Электрокинетические преобразователи информации (Энергия, M., 1973).

[5] Б.П. Шарфарец, Науч. приборостроение, 29 (2), 103 (2019).

[6] С.С. Духин, Б.В. Дерягин, Электрофорез (Наука, М., 1986).

[7] В.И. Ролдугин, Физикохимия поверхности (Интеллект, Долгопрудный, 2011).

[8] Е.Д. Щукин, А.В. Перцов, Е.А. Амелина, Коллоидная химия (Высш. шк., М., 2004).

[9] А.Н. Гузь, Введение в динамику сжимаемой вязкой жидкости (LAP Lambert Publ., Saarbrucken, 2017).

[10] Физическая энциклопедия (БРЭ, М., 1992), т. 3. 